\title{
Analysis of the Occlusal Stress Transmitted to the Inferior Alveolar Nerve by an Osseointegrated Threaded Fixture
}

\author{
G. Sammartino, ${ }^{*}$ G. Marenzi, ${ }^{*}$ R. Citarella,${ }^{\dagger}$ R. Ciccarelli, ${ }^{\ddagger}$ and H-L Wang ${ }^{\S}$
}

Background: Altered sensation can occur after the placement or loading of mandibular implants. Limited evidence exists with regard to the proper distance between the implant and the mandibular nerve to ensure the nerve's integrity and physiologic activity. The proper distance should come from evaluation of clinical data as well as from biomechanical analyses.

Methods: A numeric mandibular model based on the boundary element method was created to simulate a mandibular segment containing a threaded fixture so that the pressure on the trigeminal nerve, as induced by the occlusal loads, could be assessed. Such pressure distributions were evaluated with different distances of the fixture from the mandibular canal and considering different bone densities. Although all simulations considered a canal that was orthogonal to the implant axis, in one case, the effects of an inclined canal were analyzed.

Results: The nerve pressure increased rapidly with a bone density decrease. A low mandibular cortical bone density caused a major nerve pressure increase.

Conclusion: Our study suggested a distance of $1.5 \mathrm{~mm}$ to prevent implant damage to the underlying inferior alveolar nerve when biomechanical loading was taken into consideration. J Periodontol 2008;79:1735-1744.

\section{KEY WORDS}

\section{Dental implants; inferior alveolar nerve.}

\footnotetext{
* Section of Oral Surgery, Department of Odontostomatological and Maxillofacial Sciences, University of Naples "Federico II," Naples, Italy.

$\dagger$ Department of Mechanical Engineering, University of Salerno, Fisciano, Italy.

\# Department of Radiology and Radiotherapy, University of Naples "Federico II."

$\S$ Department of Periodontics and Oral Medicine, University of Michigan, Ann Arbor, MI.
}

$\tau$ mplant therapy has been considered one of the standard options for the rehabilitation of edentulous posterior areas. Correct presurgical planning is required to guide proper implant placement (e.g., position, diameter, and length) to avoid damage to anatomic structures, such as the maxillary sinus and mandibular canal. ${ }^{1}$ Many studies showed the possibility of trigeminal (e.g., inferior alveolar nerve) sensitivity alterations after mandibular surgical treatment ${ }^{2-5}$ and implant placement.6-12 Implant placement can cause an insult to the nervous structure and lead to transitory or irreversible alterations of inferior nerve functionality. ${ }^{8}$ Due to compression on the nerve, paresthesia and dysesthesia following implant loading have been reported. ${ }^{13}$ To prevent this complication, a correct assessment of the mandibular canal position and suitable implant size and positioning are needed. ${ }^{14,15}$ A study ${ }^{16}$ suggested the favorable positioning of a fixture with respect to adjacent natural teeth or, in more complex rehabilitations, the distance between fixtures to get an optimal distribution of occlusal forces and the best esthetic result. However, limited evidence exists with regard to the proper distance from the implant to the mandibular nerve to ensure the nerve's integrity and physiologic activity. It is our opinion that the proper distance should be determined from the evaluation of clinical data (retrospective study) as

doi: 10.1902/jop.2008.080030 
well as from biomechanical analyses. Therefore, a numeric mandibular model was created to simulate a mandibular segment containing a fixture, so that the mechanical stresses on the mandibular canal induced by the occlusal load could be assessed.

\section{MATERIALS AND METHODS}

From June to December 2007 at the University of Naples "Federico II," 123 mandibular computed tomography (CT) scans of patients between 22 and 67 years of age were examined. From each CT scan, it was possible to determine the size and bone density of the anatomic structures (cortical bone, cancellous bone, and cortical bone surrounding the mandibular canal) to be used in a numeric model. The bone density used in this study was derived based on the Hounsfield values obtained from the postprocessing of CT images. The average density and dimensional value of each examined anatomic structure was identified and reproduced in this simulated model (Table 1).

The mandibular numeric model was created using a commercial codell based on the boundary element method (BEM), a numeric methodology well suited for elastic-static analysis. The implant considered was a conic, threaded fixture with a diameter of $4.5 \mathrm{~mm}$ and a length of $11 \mathrm{~mm}$.

BEM methodology, ${ }^{17}$ even if less versatile than the finite element method (FEM) for non-linear analyses, is more accurate for linear analysis, especially in the area of a complex geometry like the threaded implant, where strong stress gradients are to be captured, or near an area undergoing high pressure, such as around the thread of an implant. Moreover, it is easier to mesh complex geometries, like the thread of the implant body, with BEM than with FEM; in most FEM studies, these are not represented as continuous hel-

\section{Table I.}

\section{Parameters of BEM Numeric Model of the Mandibular Segment}

Average thickness of the
mandibular cortical bone
Average thickness of
the cortical bone
surrounding the
mandibular channel
Average density of the
mandibular bone $\quad$ Mandibular bony cortical: $900 \mathrm{~m}$
Average diameter of
the mandibular channel medullar: 350 to $400 \mathrm{U}$

ical characteristics but are approximated as axialsymmetric independent rings. ${ }^{18}$

The mandibular segment was modeled, in a linear elastic analysis, with a mesh of $\sim 3,000$ linear elements, with the fixture connected to a prosthesis abutment on which the axial and lateral loads were applied (Fig. 1).

It is widely recognized that it is better to model the material constants of bone as orthotropic ${ }^{19}$ rather than isotropic (as done in many FEM analyses ${ }^{18}$ ), so the cortical and cancellous bone were modeled as transversely isotropic. The elastic behavior of transversely isotropic materials can be fully characterized by five elastic moduli: $E_{1}, E_{3}, v_{12}, v_{31}$, and $G_{31}$, with the remaining moduli obtained from $\mathrm{E}_{1}=\mathrm{E}_{2}, v_{23}=v_{13}=$ $v_{31} * \mathrm{E}_{1} / \mathrm{E}_{3}, \mathrm{G}_{12}=\mathrm{E}_{2} /\left(2\left[1+v_{12}\right]\right), \mathrm{G}_{23}=\mathrm{G}_{31}$. The values adopted in this work are listed in Table 2.

The trigeminal nerve was modeled as isotropic with Young modulus $\mathrm{E}=1.3 \mathrm{MPa}$ and Poisson ratio $v=0.4$. The metallic implant parts were clearly modeled as isotropic with $\mathrm{E}=120,000 \mathrm{MPa}$ and $v=0.3$.

To calculate the pressure on the nerve, a non-linear $B E M$ contact analysis was performed (for these kinds of contact analyses BEM performs better than FEM) with a null clearance imposed between the nerve and the surrounding canal structures (this is the worst case because, in reality, a minimum clearance is generally available for the nerve in the canal).

The applied loads were, alternatively, a load equal to $300 \mathrm{~N}$ along the implant axis or a lateral load (in the vestibular-lingual direction) equal to $150 \mathrm{~N}$ (Fig. 1). The choice of these forces corresponds to physiologic occlusal loads during chewing and swallowing. ${ }^{20}$

The pressure distribution induced on the underlying nervous structure was evaluated against different distances of the fixture from the mandibular canal $(\mathrm{d} 1=$ $1.0 \mathrm{~mm}$; d2 = $1.5 \mathrm{~mm}$ ).

A sensitivity analysis was also done to show the effect of bone density variations on the nerve pressure levels and, in particular, a reduction to $50 \%$ of the average value of bone density was considered. Such variations were applied simultaneously to all anatomic structures considered (cortical and spongy bone of mandible) and separately to the single parts to understand the single contributions to the fixture load absorption.

The variations in bone stiffness (E) corresponding to the aforementioned variations in bone density (d) (needed to calculate the input material properties for the numeric simulations) were calculated considering a cubic dependence of stiffness versus density ${ }^{21}(\mathrm{E}=$ $a \times d^{3}$, where $a$ is a constant).

Moreover, although all simulations considered a canal that was orthogonal to the implant axis, in one case the effects of an inclined canal were analyzed (Fig. 2).

|| BEASY ver. 10, Ashurst Lodge, Ashurst, Southampton, U.K. 

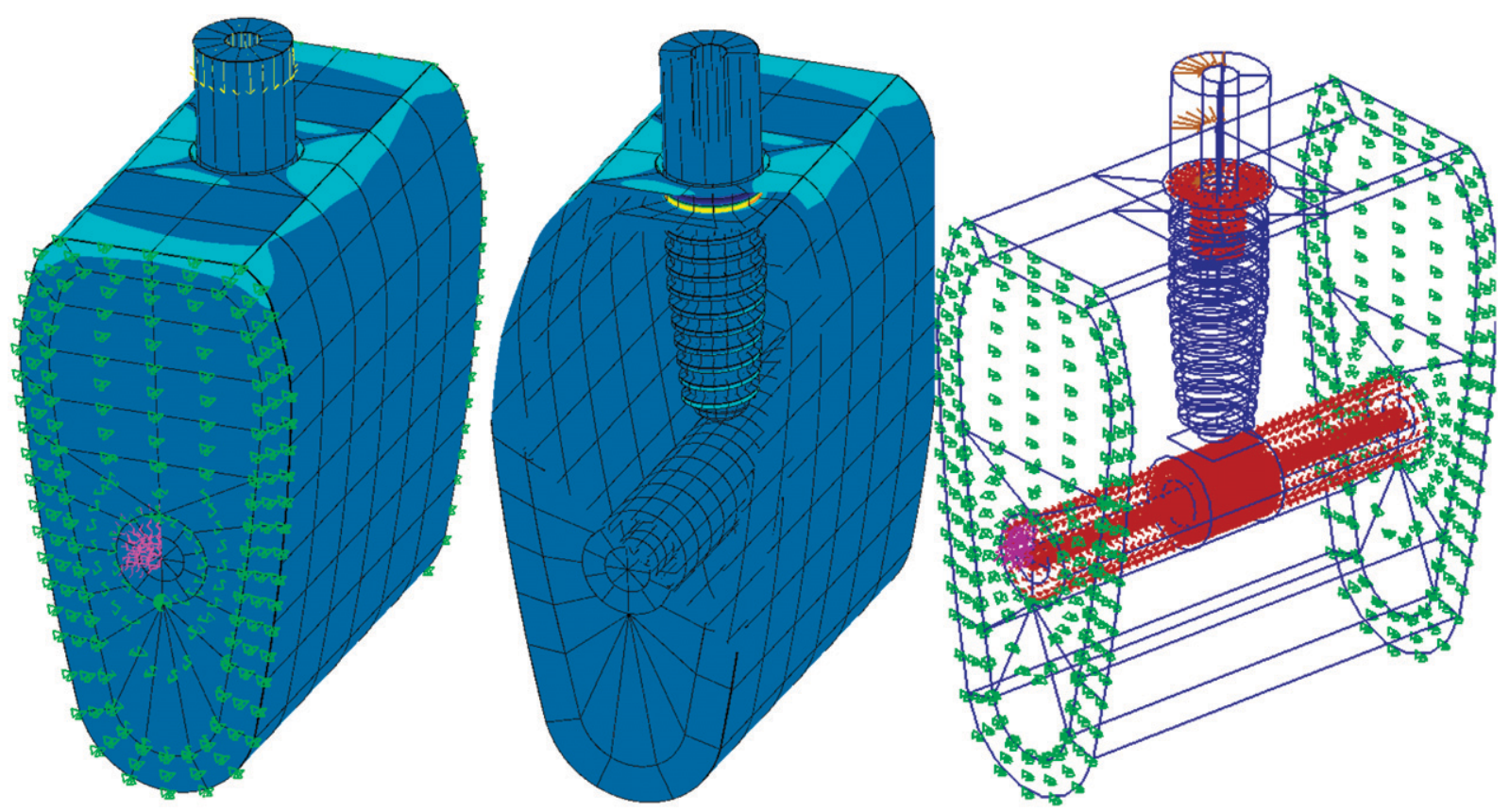

Figure I.

BEM numeric model of the mandibular segment and implant with the axial (left) and lateral (right) applied loads, mesh, and boundary conditions; internal view of the mandibular segment (middle).

\section{Table 2.}

\section{Material Properties of the Anatomic Parts Under Hypothesis of Average Bone Density or Reduced Bone Density}

\begin{tabular}{lcccccc}
\hline & \multicolumn{3}{c}{ Average Bone Density } & \multicolumn{2}{c}{ Low Bone Density (nearly half of the average density) } \\
\cline { 2 - 7 } & Canal & Spongy Bone & Cortical Bone & Canal & Spongy Bone & Cortical Bone \\
\hline $\mathrm{E}_{1}(\mathrm{MPa})$ & $2.03 \mathrm{E}+03$ & $7.19 \mathrm{E}+02$ & $1.22 \mathrm{E}+04$ & $2.03 \mathrm{E}+02$ & $7.19 \mathrm{E}+01$ & $1.22 \mathrm{E}+03$ \\
$\mathrm{E}_{3}(\mathrm{MPa})$ & $3.20 \mathrm{E}+03$ & $1.14 \mathrm{E}+03$ & $1.93 \mathrm{E}+04$ & $3.20 \mathrm{E}+02$ & $1.14 \mathrm{E}+02$ & $1.93 \mathrm{E}+03$ \\
$\mathrm{G}_{23}(\mathrm{MPa})$ & $7.24 \mathrm{E}+02$ & $2.55 \mathrm{E}+02$ & $4.37 \mathrm{E}+03$ & $7.24 \mathrm{E}+01$ & $2.55 \mathrm{E}+01$ & $4.37 \mathrm{E}+02$ \\
$\nu_{31}$ & 0.364 & 0.368 & 0.366 & 0.364 & 0.368 & 0.366 \\
$\nu_{12}$ & 0.341 & 0.342 & 0.345 & 0.341 & 0.342 & 0.345 \\
\hline
\end{tabular}

\section{RESULTS}

In Figure 3, the BEM contour plot shows the pressure on the nervous structure with an average bone density (as provided by the CTs) for all anatomic components: at a distance of $1.5 \mathrm{~mm}$ between the fixture and the upper part of the mandibular channel, the axial load produced a negligible maximum pressure on the nerve $(>40 \mathrm{~mm} \mathrm{Hg})$. Conversely, the pressure increased rapidly when the bone density decreased: considering a decrease of bone density to $50 \%$ of the average value for all anatomic components, the maximum pressure value on the nerve increased almost 10 times, reaching $347 \mathrm{~mm} \mathrm{Hg}$ (Fig. 4).

To understand which part is critical for the fixture load absorption, further simulations were done with the bone weakening affecting only some of the anatomic structures and keeping the fixture-canal distance at $1.5 \mathrm{~mm}$.

Weakening of the cortical bone around the canal and of spongy bone resulted in a moderate increase of the nerve pressure from $\mathrm{P}_{\max }$ (the maximum pressure on the considered area) $=40 \mathrm{~mm} \mathrm{Hg}$ to $\mathrm{P}_{\max }=99 \mathrm{~mm}$ $\mathrm{Hg}$ (Fig. 5). On the contrary, weakening of the mandibular spongy bone resulted in a decrease of the 
maximum nerve pressure to $25 \mathrm{~mm} \mathrm{Hg}$ (Fig. 6), showing that a softer spongy bone is not able to transfer a high load to the canal. Weakening of just the canal bone (Fig. 7) or just the mandibular cortical bone (Fig. 8) produced a moderate increase in nerve pressure: $p_{\max }=68 \mathrm{~mm} \mathrm{Hg}$ and $p_{\max }=53 \mathrm{~mm} \mathrm{Hg}$, respectively. Another combination considered was reduction of the density of the mandible and canal cortical bone: a stiff spongy bone is more effective in transferring occlusal loads to the underlying canal, this is because of a non-negligible pressure increase on the nerve to $p_{\max }=109 \mathrm{~mm} \mathrm{Hg}$ (Fig. 9). The last combination analyzed was related to low density for all anatomic components and an added total cortical resorption around the fixture: this was the worst condition and resulted in the greatest increase in nerve pressure ( $p_{\max }=388 \mathrm{~mm} \mathrm{Hg}$; (Fig. 10).

With a reduced bone density for all anatomic parts, if the canal is not perpendicular to the axially loaded fixture but is inclined as in Figure 2 and with the central part $1.5 \mathrm{~mm}$ from the fixture, the maximum pressure on the nerve is $355 \mathrm{~mm} \mathrm{Hg}$ (Fig. 11), showing that a moderate inclination does not significantly change the pressure on the nerve.

In the previous configuration (inclined canal), a lateral load of $150 \mathrm{~N}$ did not produce any significant pressure $\left(p_{\max }=10 \mathrm{~mm} \mathrm{Hg}\right.$ ) on the inferior alveolar nerve in case of low density for all structures (Fig. 12); clearly, this also holds true in case of average bone density.

With all anatomic components affected by a bone density reduction, if the distance between the fixture and the canal was decreased to $1 \mathrm{~mm}$, the maximum nerve pressure was $356 \mathrm{~mm} \mathrm{Hg}$ (Fig. 13), showing that if the initial condition was judged critical with reference to the nerve pressure, increasing the fixture-canal distance by $0.5 \mathrm{~mm}$ (to $1.5 \mathrm{~mm}$ ) did not seem to provide an additional safety margin.

On the contrary, the dependence of nerve pressure on the fixture-canal distance becomes relevant if considering average values for bone density.
Figure 2.

BEM numeric model of the mandibular canal and conic-shaped fixture when modeled as being perpendicular (left) or inclined (right).
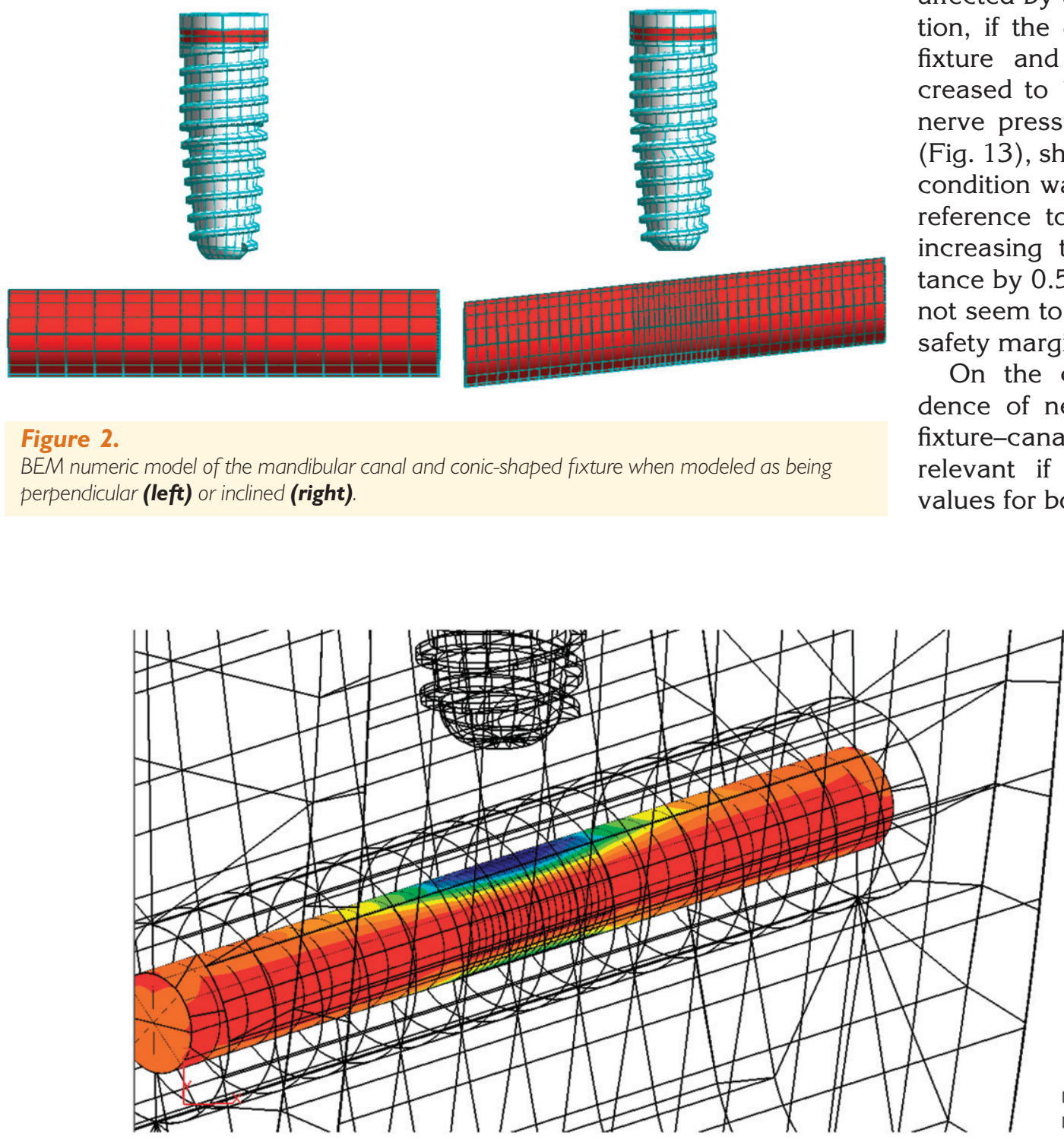

$1.07289 \mathrm{e}-005$

$-4.22044 \mathrm{e}-004$

$-8.54816 \mathrm{e}-004$

$-1.28759 \mathrm{e}-003$

$-1.72036 \mathrm{e}-003$

$-2.15313 \mathrm{e}-003$

$-2.58590 \mathrm{e}-003$

$-3.01868 \mathrm{e}-003$

$-3.45145 \mathrm{e}-003$

$-3.88422 \mathrm{e}-003$

$-4.31699 \mathrm{e}-003$

$-4.74976 \mathrm{e}-003$

Normal Traction $\operatorname{Max}=5.93054 \mathrm{E}-05$ $\operatorname{Min}=-4.79834 \mathrm{E}-03$

Figure 3.

Pressure $\left(\mathrm{N} / \mathrm{mm}^{2}\right)$ on the inferior alveolar nerve, under fixture axial load, for an average bone density and a fixture-canal distance equal to $1.5 \mathrm{~mm}$. 


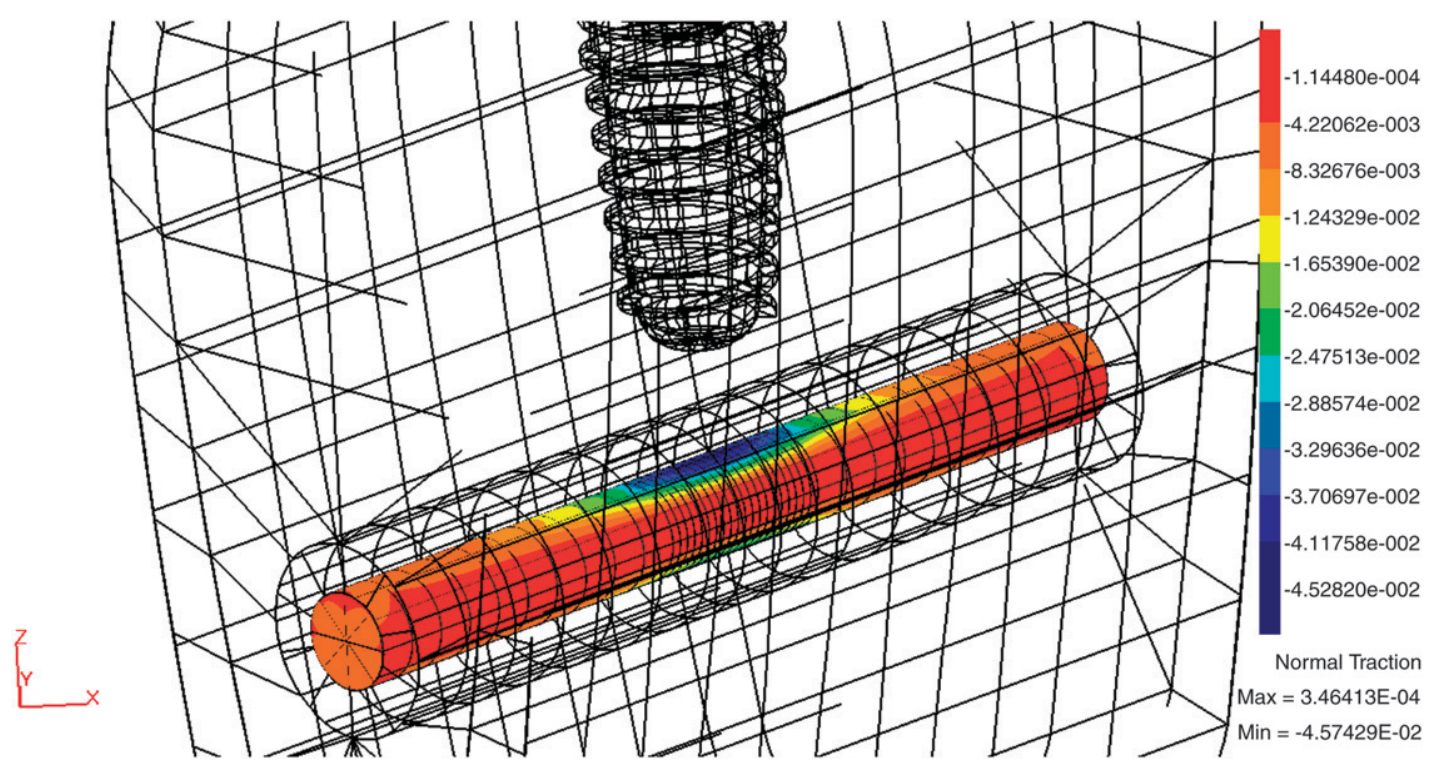

Figure 4.

Pressure $\left(\mathrm{N} / \mathrm{mm}^{2}\right)$ on the inferior alveolar nerve, under fixture axial load, for a reduced bone density and a fixture-canal distance equal to $1.5 \mathrm{~mm}$.

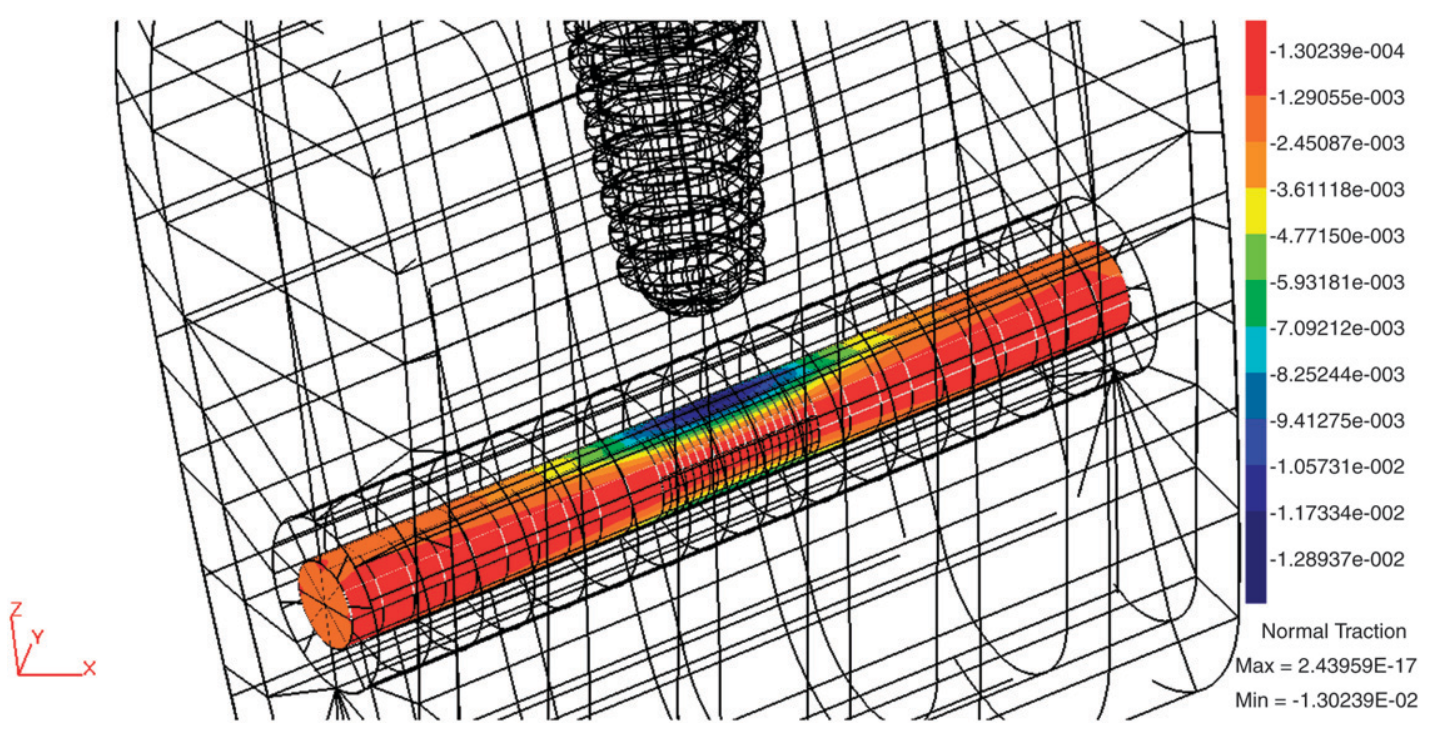

Figure 5.

Pressure $\left(\mathrm{N} / \mathrm{mm}^{2}\right)$ on the inferior alveolar nerve, under fixture axial load, with reduced density (affecting only cortical bone surrounding the canal and mandible spongy bone) and a fixture-canal distance equal to $1.5 \mathrm{~mm}$.

\section{DISCUSSION}

Studies ${ }^{6-12}$ reported mandibular nerve sensitivity alteration following implant placement. This alteration can be transitory or non-reversible, depending on the severity of nerve damage. ${ }^{8}$ Functional alteration of the mandibular nerve can be caused by an excessive drilling pressure or a soft tissue injury during the surgical phase. Also, a post-surgery edema or hema- toma and a real contact of the implant to the mandibular nerve can be responsible for sensitivity alterations. ${ }^{2-5}$

When the fixture is placed too close to the mandibular canal, it can induce a mechanical stress on the underlying canal, resulting in impairment of nerve function. ${ }^{13}$ Some retrospective studies $8,12,14$ suggested that a minimum distance is needed to 


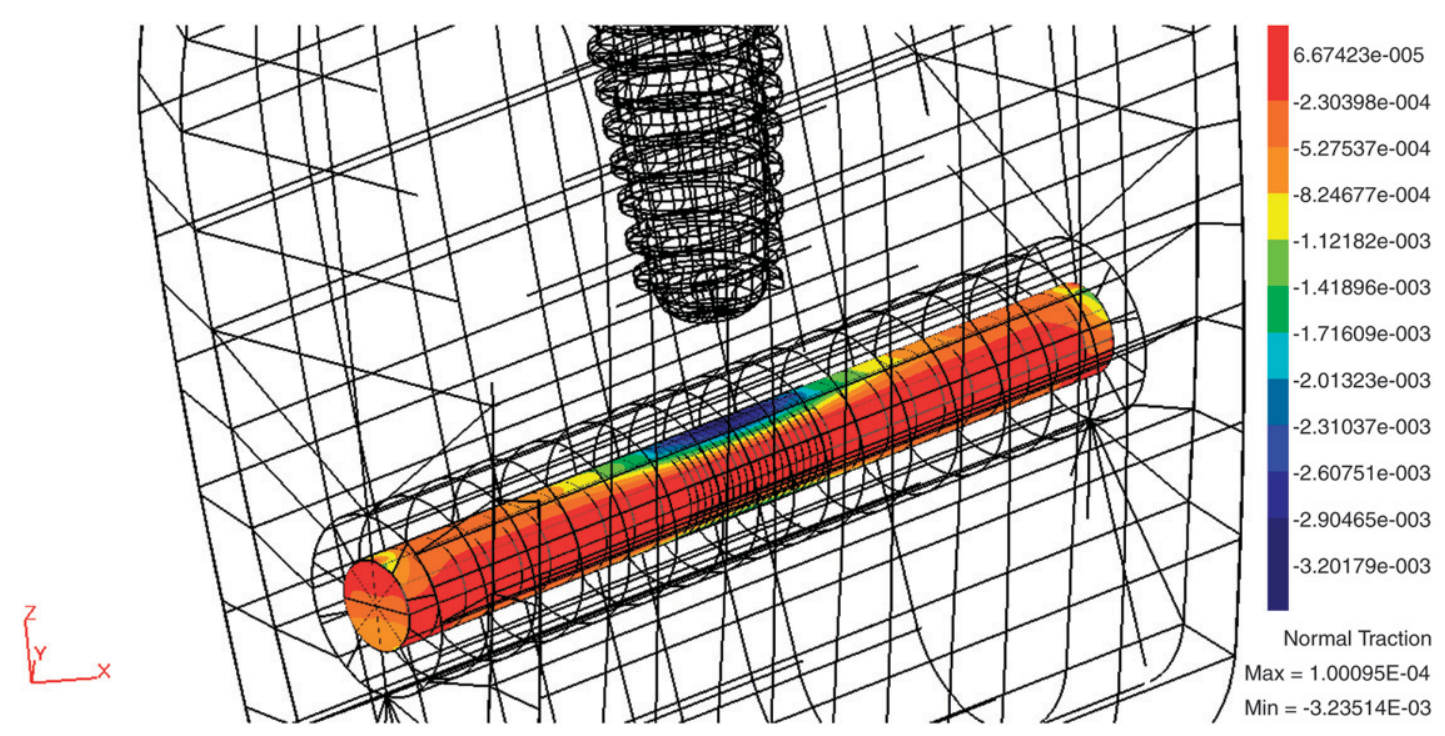

Figure 6.

Pressure $\left(\mathrm{N} / \mathrm{mm}^{2}\right)$ on the inferior alveolar nerve, under fixture axial load, with reduced density (affecting only the spongy bone) and a fixture-canal distance equal to $1.5 \mathrm{~mm}$.

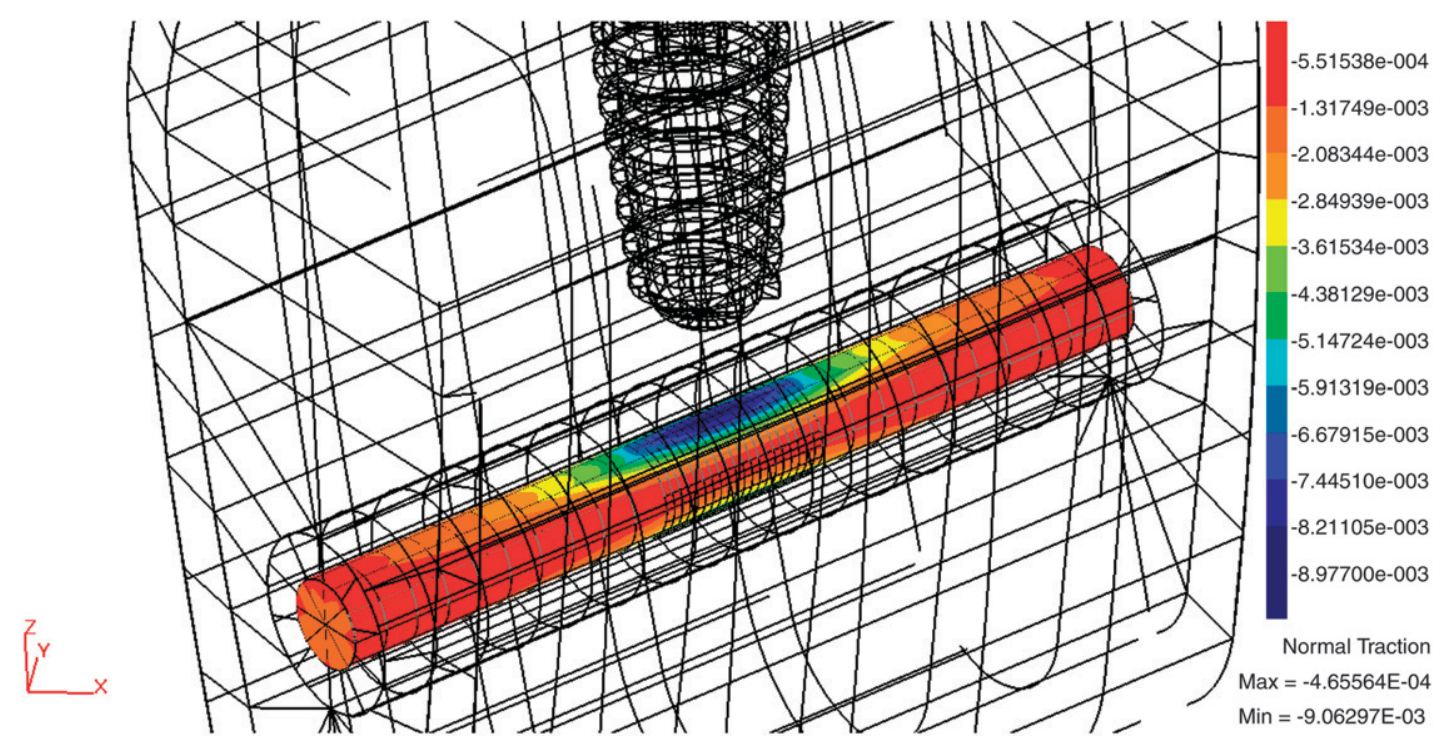

Figure 7.

Pressure $\left(\mathrm{N} / \mathrm{mm}^{2}\right.$ ) on the inferior alveolar nerve, under fixture axial load, with reduced density (affecting only the cortical bone around the mandibular canal) and a fixture-canal distance equal to $1.5 \mathrm{~mm}$.

avoid nerve damage; however, those studies did not take the biomechanical aspect into consideration.

Although some surgical solutions to nerve injuries have been proposed, ${ }^{13}$ it is recommended to plan the implant position before insertion to ensure a safe distance between the fixture and the mandibular canal.
In neurosurgery, the compressive technique is performed with stents and applied transcutaneously, close to the oval foramen, to control neuralgias of the trigeminal nerve. ${ }^{22-27}$ Pressures $>100$ to 200 $\mathrm{mm} \mathrm{Hg}$ applied for 30 to 60 seconds to the trigeminal nerve can cause a block of the nervous impulse and solve the pain problem. ${ }^{22,26}$ 


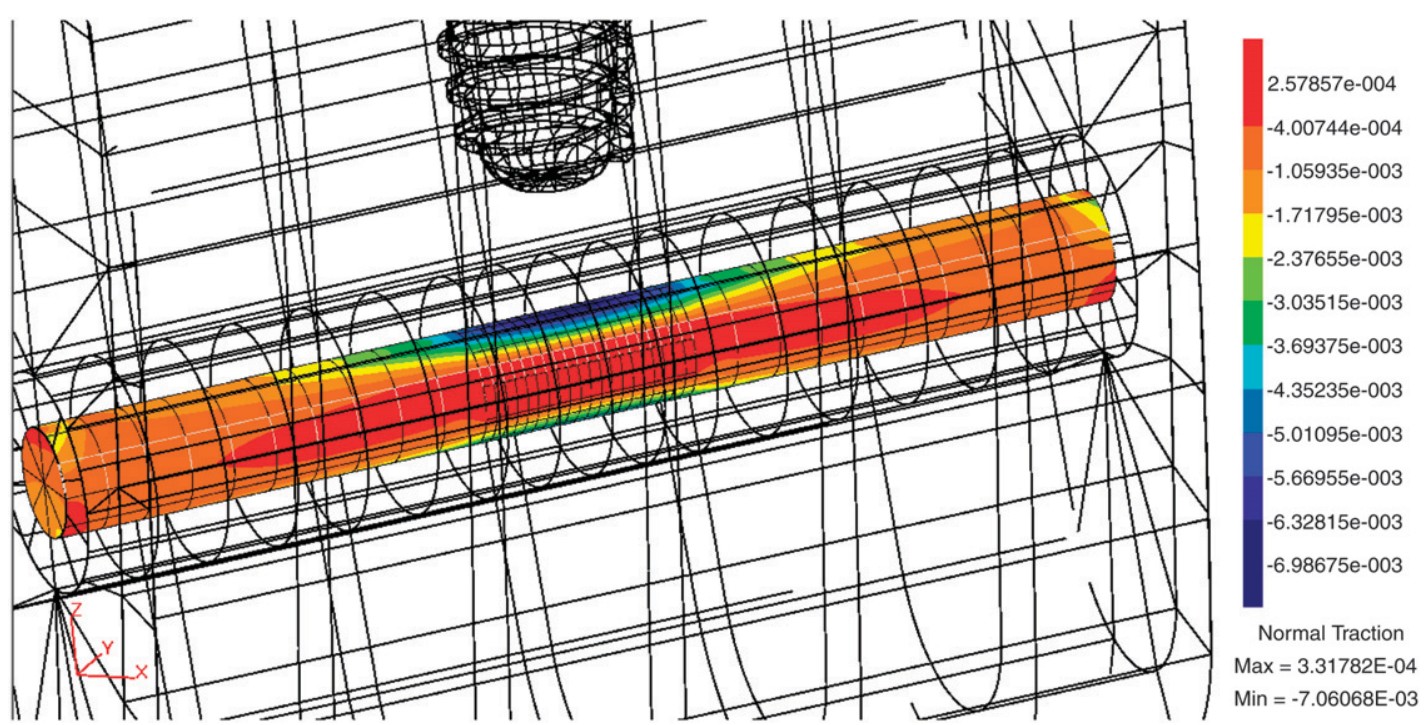

Figure 8.

Pressure $\left(\mathrm{N} / \mathrm{mm}^{2}\right.$ ) on the inferior alveolar nerve, under fixture axial load, with reduced density (affecting only the mandible cortical bone) and a fixture-canal distance equal to $1.5 \mathrm{~mm}$.

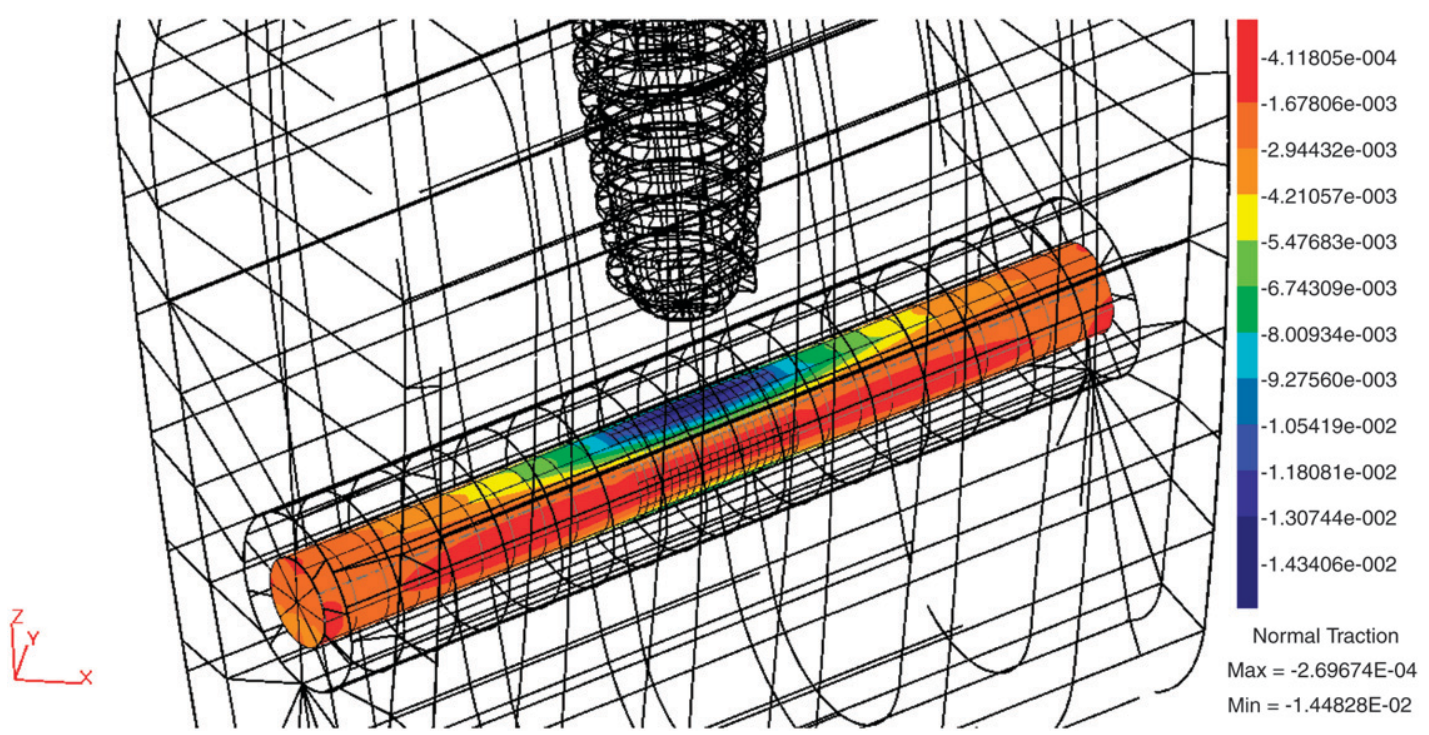

Figure 9.

Pressure $\left(\mathrm{N} / \mathrm{mm}^{2}\right)$ on the inferior alveolar nerve, under fixture axial load, with reduced density (cortical bone of mandible and canal) and a fixture-canal distance equal to $1.5 \mathrm{~mm}$.

This investigation allowed an evaluation of the pressures that a conic, threaded fixture undergoing a functional load is able to transmit to the underlying bony structures.

Data from this investigation showed the sensitivity of nerve pressure to variations in bone density of the different anatomic structures, with particular reference to a distance between the implant (bottom part) and the canal (upper part) of $1.5 \mathrm{~mm}$ : the general trend was that the nerve pressure increased with a decrease in bone density.

Moreover, the effects on the nerve of a total cortical resorption around the implant were evaluated.

The data obtained from these simulations suggested that, considering the worst condition in terms of bone density, a fixture-canal distance of $1.5 \mathrm{~mm}$ 


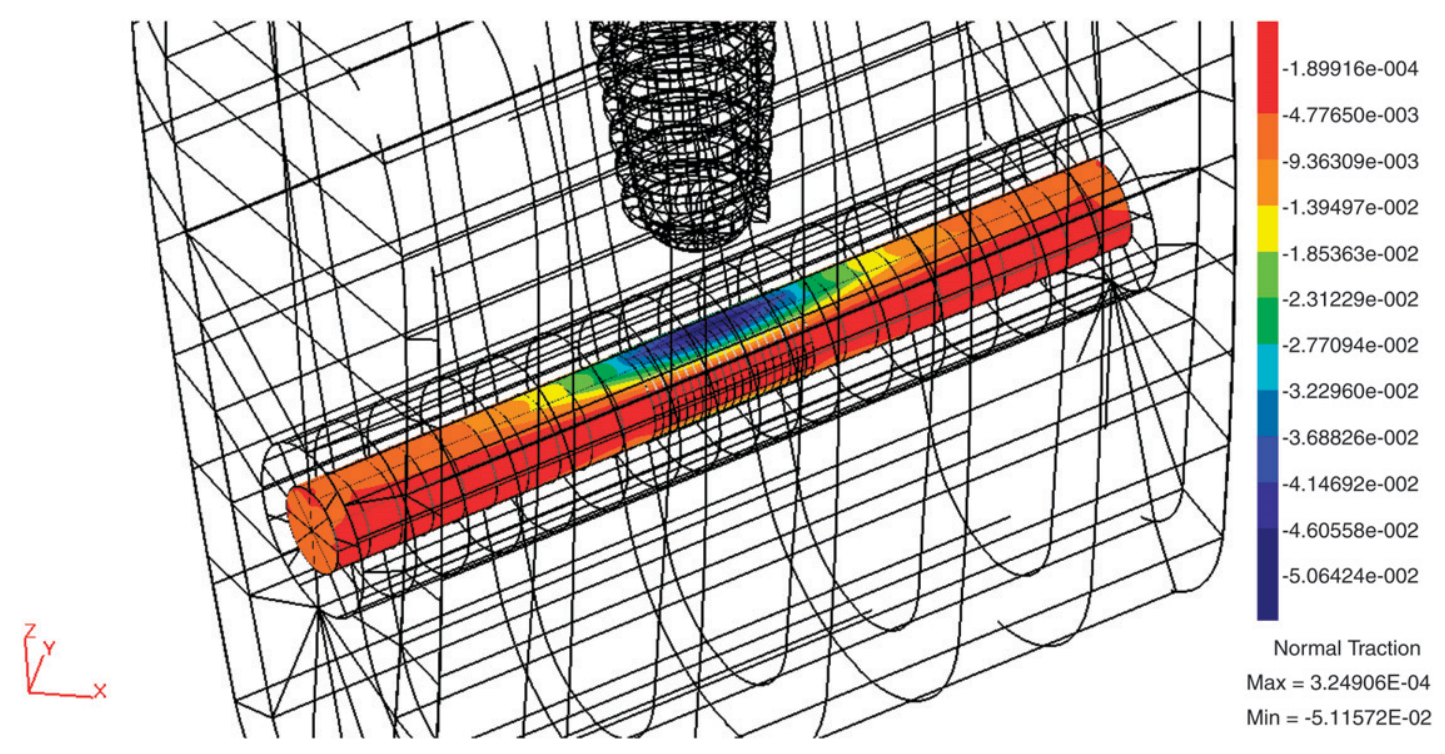

Figure 10.

Pressure ( $\left(\mathrm{N} / \mathrm{mm}^{2}\right.$ ) on the inferior alveolar nerve, under fixture axial load, with reduced density (affecting all components), total cortical bone resorption around the implant, and a fixture-canal distance equal to $1.5 \mathrm{~mm}$.

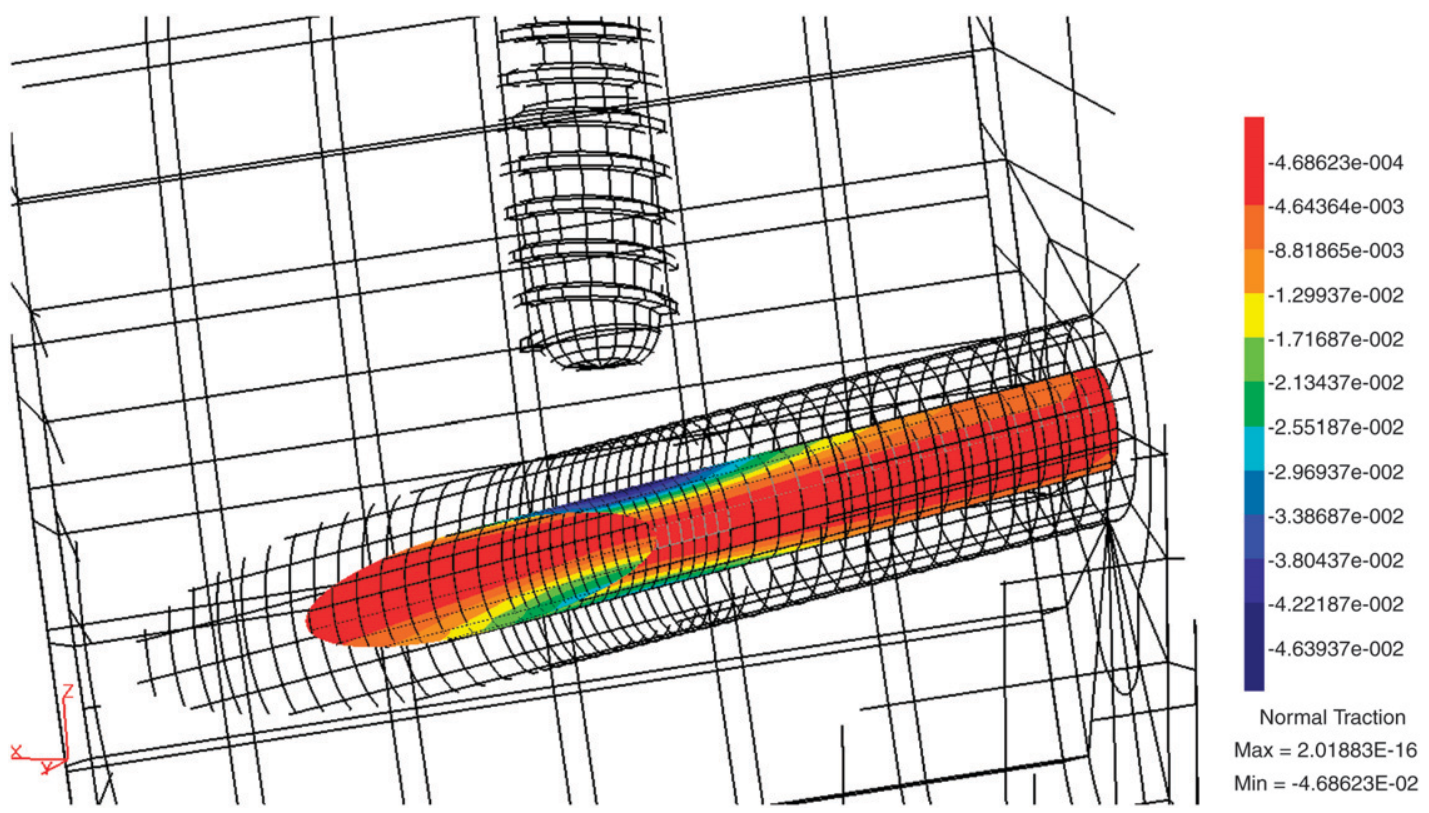

Figure II.

Pressure ( $\mathrm{N} / \mathrm{mm}^{2}$ ) on the inferior alveolar nerve, under fixture axial load and inclined canal, with reduced density (affecting all parts) and a fixture-canal distance equal to $1.5 \mathrm{~mm}$.

can prevent excessive pressure on the trigeminal nerve and consequent mandibular sensitivity alterations caused by occlusal forces transmitted by an implant.

The maximum calculated pressure values are very similar to the pressures used in neurosurgery.
Because the high force produced during occlusal contact persisted for an average of 115 milliseconds, ${ }^{19}$ it did not alter the trigeminal functionality.

This implies that $1.5 \mathrm{~mm}$ is an acceptable reference distance in presurgical planning. 


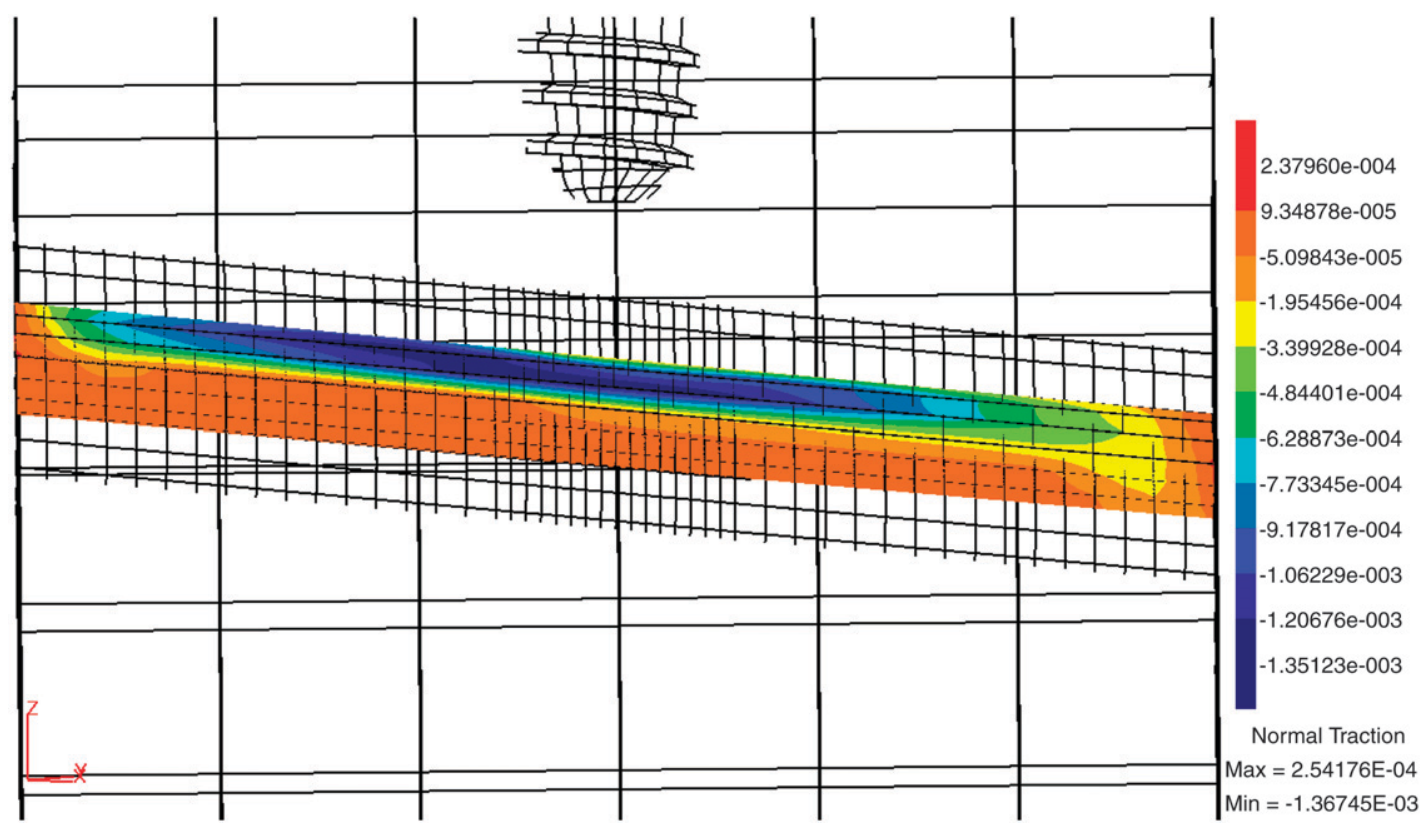

Figure 12.

Pressure $\left(\mathrm{N} / \mathrm{mm}^{2}\right)$ on the inferior alveolar nerve, under fixture lateral load and inclined canal, with reduced density and a fixture-canal distance equal to $1.5 \mathrm{~mm}$.

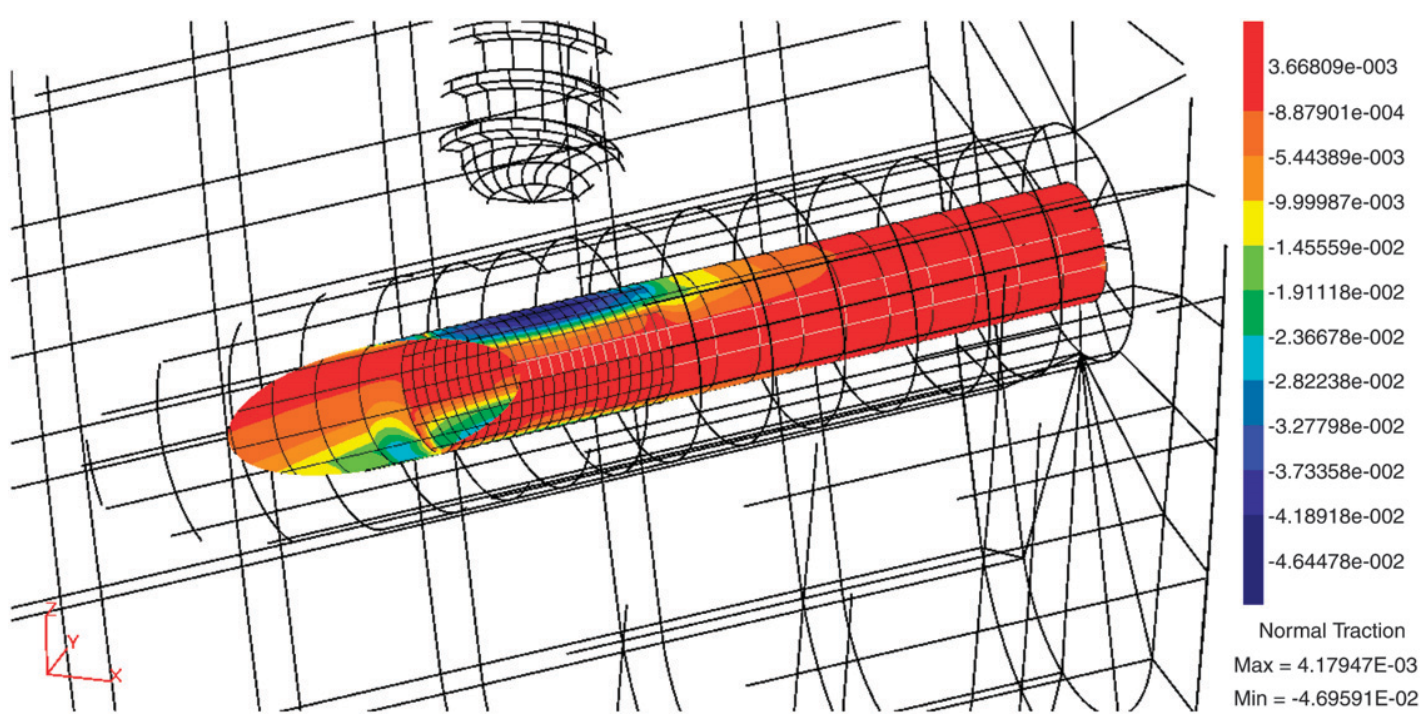

Figure 13.

Pressure ( $\mathrm{N} / \mathrm{mm}^{2}$ ) on the inferior alveolar nerve, under fixture axial load and perpendicular canal, with reduced density (affecting all parts) and a fixture-canal distance equal to $1 \mathrm{~mm}$.

\section{ACKNOWLEDGMENT}

The authors have no financial interest in any company or any of the products mentioned in this article.

\section{REFERENCES}

1. Kopp KC, Koslow AH, Abdo OS. Predictable implant placement with a diagnostic/surgical template and advanced radiographic imaging. J Prosthet Dent 2003; 89:611-615.

2. De Koomen HA. A prosthetic view on vestibuloplasty with free mucosal graft. Int J Oral Surg 1977;6:3841.

3. Bailey PH, Bays RA. Evaluation of long-term sensory changes following mandibular augmentation procedures. J Oral Maxillofac Surg 1984;42:722-727. 
4. Kipp DP, Goldstein BH, Weiss WW Jr. Dysesthesia after mandibular third molar surgery: A retrospective study and analysis of 1,377 surgical procedures. J Am Dent Assoc 1980;100:185-192.

5. Simpson HE. Injuries to the inferior dental and mental nerves. J Oral Surg 1958;16:300-305.

6. Walton JN. Altered sensation associated with implants in the anterior mandible: A prospective study. J Prosthet Dent 2000;83:443-449.

7. Ellies LG. Altered sensation following mandibular implant surgery: A retrospective study. $J$ Prosthet Dent 1992;68:664-671.

8. Wismeijer D, van Waas MA, Vermeeren JI. Patients' perception of sensory disturbances of the mental nerve before and after implant surgery: A prospective study of 110 patients. Br J Oral Maxillofac Surg 1997;35: 254-259.

9. Rubenstein JE, Taylor TD. Apical nerve transection resulting from implant placement: A 10-year follow-up report. J Prosthet Dent 1997;78:537-541.

10. Ellies LG, Hawker PB. The prevalence of altered sensation associated with implant surgery. Int $J$ Oral Maxillofac Implants 1993;8:674-679.

11. Gregg JM. Neuropathic complications of mandibular implant surgery: Review and case presentations. Ann R Australas Coll Dent Surg 2000;15:176-180.

12. Bartling R, Freeman K, Kraut RA. The incidence of altered sensation of the mental nerve after mandibular implant placement. J Oral Maxillofac Surg 1999;57: 1408-1410.

13. Levitt DS. Apicectomy of an endosseous implant to relieve paresthesia: A case report. Implant Dent 2003;12: 202-205.

14. Greenstein G, Tarnow D. The mental foramen and nerve: Clinical and anatomical factors related to dental implant placement: A literature review. J Periodontol 2006;77:1933-1943.

15. Santler G, Kärcher H, Ruda C. Indications and limitations of three-dimensional models in cranio-maxillofacial surgery. J Craniomaxillofac Surg 1998;26:11-16.

16. Kois JC. Predictable single tooth peri-implant esthetics: Five diagnostic keys. Compend Contin Educ Dent 2001; 22:199-206.

17. Hartman F. Introduction to Boundary Elements: Theory and Applications. Berlin: Springer-Verlag; 1989:154215.
18. Bono B, Cirello A, Geraci D, Pasta A, Cassaro A. FEM model achievement for dental implant characterization. Eur J Implant Prosthodontics 2005;2:75-85.

19. Rho JY, Hobatho MC, Ashman RB. Relations of mechanical properties to density and CT numbers in human bone. Med Eng Phys 1995;17:347-355.

20. Gibbs $\mathrm{CH}$, Mahan PE, Lundeen HC, et al. Occlusal forces during chewing and swallowing as measured by sound transmission. J Prosthet Dent 1981;46:443-449.

21. Wirtz DC, Schiffers N, Pandorf T, Radermacher K, Weichert D, Forst R. Critical evaluation of known bone material properties to realize anisotropic FE-simulation of the proximal femur. J Biomech 2000;33:13251330.

22. Lee ST, Chen JF. Percutaneous trigeminal ganglion balloon compression for treatment of trigeminal neuralgia - Part 1: Pressure recordings. Surg Neurol 2003; 59:63-67.

23. Lee ST, Chen JF. Percutaneous trigeminal ganglion balloon compression for treatment of trigeminal neuralgia - Part 2: Results related to compression duration. Surg Neurol 2003;60:149-154.

24. Brown JA, Pilitsis JG. Percutaneous balloon compression for the treatment of trigeminal neuralgia: Results in 56 patients based on balloon compression pressure monitoring. Neurosurg Focus 2005;18:E10.

25. Brown JA, McDaniel MD, Weaver MT. Percutaneous trigeminal nerve compression for treatment of trigeminal neuralgia: Result in 50 patients. Neurosurgery 1993;32:570-573.

26. Brown JA, Hoeflinger B, Long PB, et al. Axon and ganglion cell injury in rabbits after percutaneous trigeminal balloon compression. Neurosurgery 1996;38: 993-1004.

27. Belber CJ, Rak RA. Balloon compression rhizolysis in the surgical management of trigeminal neuralgia. Neurosurgery 1987;20:908-913.

Correspondence: Dr. Gilberto Sammartino, Division of Oral Surgery, Department of Odontostomatological and Maxillofacial Sciences, University of Naples "Frederico II", Via Pansini 5, Ed. 14, 80131 Naples, Italy. Fax: 39-817462654; e-mail: gilberto.sammartino@unina.it.

Submitted January 15, 2008; accepted for publication March 16, 2008. 